\section{Pairs of Latin squares that produce digram-balanced Greco-Latin designs: A BASIC program}

\begin{abstract}
JAMES R. LEWIS
International Business Machines, Inc, Boca Raton, Florida

It is possible to create pairs of Latin squares that are digram balanced (in other words, that counterbalance immediate sequential effects) in a Greco-Latin design. A behavioral researcher can use these squares to design an efficient, well-balanced study without relying on chance. Researchers can apply these squares to any experiment in which they must pair conditions with different stimuli in a within-subject design. For experiments with a large number of conditions, however, the procedure is very timeconsuming if done manually. A BASIC program is described that generates the correct pairs of squares for experiments with as many as 80 conditions.
\end{abstract}

The usual Latin square design ensures that each experimental condition appears an equal number of times in each row and column of the square (Kirk, 1982; Myers, 1979). Williams (1949), Bradley (1958), and Wagenaar (1969) described Latin squares that have the property of counterbalancing immediate sequential effects. Wagenaar referred to this property as "digram-balanced." As Bradley (1958) states, this is important when:

it may be suspected that reaction to any given experimental condition will be influenced (a) by the number of experimental conditions preceding it, i.e., by its ordinal position in the sequence of presentation, (b) by the particular experimental condition immediately preceding it. This situation is commonly encountered in psychological experiments when every subject is run under all experimental conditions. Such experimental designs reduce variance at the expense of introducing sequential effects such as learning, fatigue, and interactions between reactions to various experimental conditions, particularly those adjacent in order of presentation. (p. 525)

Bradley (1958) rigorously proved a simple method of construction that guarantees this counterbalancing in a single square with an even number of conditions, and Williams (1949) provided alternative methods for this counterbalancing in two squares when the number of conditions is odd. With a variation on these methods of construction, Lewis (1989) published algorithms for creating pairs of Latin squares that simultaneously counterbalance both immediate sequential effects and the pairing of conditions and stimuli for within-subject experiments.

I would like to acknowledge the helpful comments from Jerome $\mathrm{L}$. Myers and an anonymous reviewer, which substantially improved this paper. Correspondence should be addressed to James R. Lewis, Design Center/Human Factors Department, IBM Corp., P.O. Box 1328, Boca Raton, FL 33429-1328.
Across these pairs of Latin squares, the stimuli and conditions form a Greco-Latin design (Kirk, 1982) in which each level of the condition and each level of the stimulus appear exactly once in each row and column of each square, and each combination appears exactly twice over both squares. Across both squares, each condition immediately precedes and follows each other condition exactly twice, and each stimulus immediately precedes and follows each other stimulus exactly twice, forming a digram-balanced design.

Such experiments are very common in human factors research. For example, a behavioral researcher could use these squares to design an experiment to investigate the perceived legibility of different computer displays. Participants in the study would read different text samples on the displays and would rank the displays along the dimension of perceived legibility. Using Lewis's (1989) algorithms, a study with five displays and five text samples would require a minimum of 10 participants to complete a digram-balanced Greco-Latin design.

\section{The Program}

The IBM ${ }^{1}$ BASIC program LATBUILD uses Lewis's (1989) algorithms to create the pairs of squares. The only program inputs are the number of conditions in the experiment and a name for the output file. If the user does not provide a name for the output file, then the program's default output file name is PAIRSQUR. (Each time the program creates an output file, it will overwrite any existing file with the same name.) The output file contains an ASCII listing of the pairs of conditions and stimuli in an easy-to-read format. The user can either print the file or can view it with any standard editor or word processor. Figure 1 shows the output for five conditions, and Figure 2 shows the output for six conditions.

\section{Discussion}

After the program generates the squares, the next step is to randomly assign conditions and stimuli to the numbers in the squares. The appropriate method of analysis depends on the nature of the data that the researcher collects in the experiment. Three potential methods are repeated measures analysis of variance (Kirk, 1982; Myers, 1979), multivariate analysis of variance (Cliff, 1987), and the Friedman two-way layout test (Hollander \& Wolfe, 1973).

A behavioral researcher can use these squares to design an efficient, well-balanced study without relying on chance assignment. Researchers can apply these squares to any experiment in which they must pair conditions with different stimuli in a within-subject design. Because the program in this paper can create the correct pairs of squares easily for experiments with as many as 80 conditions, this formerly time-consuming procedure is now easier to apply. 
Across both squares, the design is simultaneously digram balanced and Greco-Latin. However, the characteristics of the individual squares are different depending on whether the number of experimental conditions is even or odd. When the number of conditions is odd, each square is Greco-Latin because the condition-stimulus pairing is balanced within the square (although a single square is not digram balanced). When the number of conditions is even, the squares are not Greco-Latin because the condition-stimulus pairing is not balanced within a single square (although a single square is digram balanced).

Researchers can use the program output in a number of other ways. If a researcher only needs digram-balanced designs, without considering condition-stimulus pairing, then he/she can just ignore the stimulus output. For an even number of conditions only, researchers can use the program to produce two different Latin squares that are each digram balanced. For an odd number of conditions only, researchers can use the program to produce two different Greco-Latin squares.

\section{Program Availability}

The source code for this program may be obtained by sending a DOS-formatted 3.5-in. disk to the author.

\begin{tabular}{|c|c|c|c|c|c|}
\hline \multirow{4}{*}{$\begin{array}{l}\text { Subiect } \\
1 \text { Condition: } \\
\text { Stimulus: }\end{array}$} & \multicolumn{2}{|c|}{ Order } & \multirow[b]{2}{*}{3} & \multirow[b]{2}{*}{4} & \multirow[b]{2}{*}{5} \\
\hline & 1 & 2 & & & \\
\hline & $\overline{1}$ & 5 & 2 & $\overrightarrow{4}$ & \\
\hline & 3 & 4 & 2 & 5 & 1 \\
\hline 2 Condition: & 2 & 1 & 3 & 5 & \\
\hline Stimulus: & 4 & 5 & 3 & 1 & \\
\hline 3 Condition: & 3 & 2 & 4 & 1 & \\
\hline Stimulus: & 5 & 1 & 4 & 2 & \\
\hline 4 Condition: & 4 & 3 & 5 & 2 & \\
\hline Stimulus: & 1 & 2 & 5 & 3 & \\
\hline 5 Condition: & 5 & 4 & 1 & 3 & \\
\hline Stimulus: & 2 & 3 & 1 & 4 & \\
\hline 6 Condition: & 3 & 4 & 2 & 5 & \\
\hline Stimulus: & 1 & 5 & 2 & 4 & \\
\hline 7 Condition: & 4 & 5 & 3 & 1 & \\
\hline Stimulus: & 2 & 1 & 3 & 5 & \\
\hline 8 Condition: & 5 & 1 & 4 & 2 & \\
\hline Stimulus: & 3 & 2 & 4 & 1 & \\
\hline 9 Condition: & 1 & 2 & 5 & 3 & \\
\hline Stimulus: & 4 & 3 & 5 & 2 & \\
\hline $10 \mathrm{Cor}$ & 2 & 3 & 1 & 4 & \\
\hline Stimulus: & 5 & 4 & 1 & 3 & \\
\hline
\end{tabular}

Figure 1. LATBUILD program output file for five conditions.

\begin{tabular}{cllllll} 
& Order & & & & \\
Subject & 1 & 2 & 3 & 4 & 5 & 6 \\
\hline 1 Condition: & 1 & 6 & 2 & 5 & 3 & 4 \\
Stimulus: & 1 & 2 & 6 & 3 & 5 & 4 \\
2 Condition: & 2 & 1 & 3 & 6 & 4 & 5 \\
Stimulus: & 2 & 3 & 1 & 4 & 6 & 5 \\
3 Condition: & 3 & 2 & 4 & 1 & 5 & 6 \\
Stimulus: & 3 & 4 & 2 & 5 & 1 & 6 \\
4 Condition: & 4 & 3 & 5 & 2 & 6 & 1 \\
Stimulus: & 4 & 5 & 3 & 6 & 2 & 1 \\
5 Condition: & 5 & 4 & 6 & 3 & 1 & 2 \\
Stimulus: & 5 & 6 & 4 & 1 & 3 & 2 \\
6 Condition: & 6 & 5 & 1 & 4 & 2 & 3 \\
Stimulus: & 6 & 1 & 5 & 2 & 4 & 3 \\
& & & & & & \\
7 Condition: & 6 & 1 & 5 & 2 & 4 & 3 \\
Stimulus: & 1 & 6 & 2 & 5 & 3 & 4 \\
8 Condition: & 1 & 2 & 6 & 3 & 5 & 4 \\
Stimulus: & 2 & 1 & 3 & 6 & 4 & 5 \\
9 Condition: & 2 & 3 & 1 & 4 & 6 & 5 \\
Stimulus: & 3 & 2 & 4 & 1 & 5 & 6 \\
11 Condition: & 4 & 5 & 3 & 6 & 2 & 1 \\
10 Condition: & 3 & 4 & 2 & 5 & 1 & 6 \\
Stimulus: & 4 & 3 & 5 & 2 & 6 & 1 \\
Stimulus: & 5 & 4 & 6 & 3 & 1 & 2 \\
& 6 & 5 & 1 & 4 & 2 & 3
\end{tabular}

Figure 2. LATBUILD program output file for six conditions.

\section{REFERENCES}

BradLey, J. V. (1958). Complete counterbalancing of immediate sequential effects in a Latin square design. Journal of the American Statistical Association, 53, 525-528.

Cliff, N. (1987). Analyzing multivariate data. San Diego, CA: Harcourt Brace Jovanovich.

Hollander, M., Wolfe, D. A. (1973). Nonparametric statistical methods. New York: Wiley.

KIRK, R. E. (1982). Experiment design: Procedures for the behavioral sciences. Monterey, CA: Brooks/Cole.

LEWIS, J. R. (1989). Pairs of Latin squares to counterbalance sequential effects and pairing of conditions and stimuli. In Proceedings of the 33rd Annual Meeting of the Human Factors Society (pp. 12231227). Santa Monica, CA: Human Factors Society.

MYERS, J. L. (1979). Fundamentals of experimental design. Boston: Allyn \& Bacon.

WAGENAAR, W. A. (1969). Note on the construction of digram-balanced Latin squares. Psychological Bulletin, 72, 384-386.

Williams, E. J. (1949). Experimental designs balanced for the estimation of residual effects of treatments. Australian Journal of Physical Sciences, A2, 149-168.

\section{NOTE}

1. IBM is a registered trademark of the International Business Machines Corporation. 